\title{
Mixed-reality prototypes to support early creative design
}

\author{
Stéphane Safin, Vincent Delfosse, Pierre Leclercq \\ LUCID-ULg : Lab for User Cognition and Innovative Design - University of Liège - Belgium \\ firstname.lastname@ulg.ac.be
}

\begin{abstract}
The domain we address is creative design, mainly architecture. Rooted in a multidisciplinary approach as well as a deep understanding of architecture and design, our method aims at proposing adapted mixed reality solutions to support two crucial activities : sketch-based preliminary design and distant synchronous collaboration in design. This chapter provides a summary of our work on a mixed-reality device, based on a drawing table (the Virtual Desktop), designed specifically to address real-life/business-focused issues. We explain our methodology, describe the two supported activities and the related users' needs, detail the technological solution we have developed and present the main results of multiple evaluation sessions. We conclude with a discussion of the usefulness of a profession-centered methodology and the relevance of mixed reality to support creative design activities.
\end{abstract}

\section{Introduction}

Cutting edge technologies, like the ones involved in mixed reality, are often designed by engineers focusing primarily on technological aspects, without directly involving the end-users. The user of the final product will often thus have to learn and to adapt to the new concepts this new technology will bring. The fact that precise user's needs are not at the heart of the initial phase of design can create a gap between these needs and the solution offered. This sometimes leads to the failure of the project, as the user may find it difficult to adapt to new ways of working.

We propose a different approach, based on a multidisciplinary way of working. Our methodology is rooted in a user-centered design approach, and based on a thorough analysis of a dedicated domain's field and market.

This methodology has been applied by the LUCID-ULg Lab in the computer aided architectural design field. Based on a deep knowledge of the architectural project workflow, and thanks to a close collaboration with "real" architects, we have identified two specific activities that would benefit from the support of mixed reality environments.

The first activity we want to support is the early design phase of a project. Despite the vast offer in CAD tools, the preferred support for this phase is still the traditional pen-and-paper. Even in domains where design constitutes only a part of the whole process (as for instance building or naval engineering, architecture, industrial design or town planning), there are great ideas that emerge from quick drawings made on the corner of a napkin! We will discuss in detail the many reasons for this situation. We come to realize that, for supporting this crucial phase, an immersive and non intrusive environment must be designed. We will present the Virtual Desktop environment, and the EsQUIsE software as solutions for this problem.

The second activity is about distant and synchronous collaborative design. Despite the many communication tools available, and the existence of some shared drawing applications, the industry has not adopted tools for such activity. Design is a very complex creative task. It can take a long time for the ideas to emerge and to mature. Collaborative design requires intimate and trustful communication, so that partners can share and discuss complex and fuzzy ideas during long creative sessions. If the communication media introduces even a small bias, it might ruin the whole process, as the different actors will spend most of their effort in making sure the information is properly transmitted instead of focusing on the creative part. We will introduce the combination of the Virtual Desktop and the SketSha software to support this activity and discuss how this immersive environment brings the distant partners as if they were in the same room.

We first present our methodology. We then explain the context of architectural design and the needs of the two considered situations. We detail the technological tools we have implemented to respond to these issues. We will then present the promising results of the multiple experiments we have conducted to validate our two immersive systems. We will discuss the issues they raise, their adequacy to the aimed situations and the way we 
envision their future. We then conclude on the usefulness of the user-centered design and the profession-centered methodology, and the potential of mixed reality paradigm to support creative design activities.

\section{Profession-centered methodology and user-centered design}

Even if the importance of the user is more widely recognized in software engineering domains, many complex IT projects are still driven primarily by technical issues. The question of the user and his/her activities may then be treated as secondary issues. This can lead to ill-adapted applications or environments. This technology-driven way of working can be contrasted with an anthropocentric way of designing mixed reality systems [34]. This approach grounds project development in the context of real activities, based on user needs identified by activity analysis.

We can distinguish several methods to involve users in the software development cycle. Each of them has several advantages and disadvantages. Norms and guidelines (see for example Bastien \& Scapin [3]) are a way to represent the users' capabilities and cognitive constraints. They are easy to use, practical and cheap, but they only represent static knowledge about human beings, and fail to take into account the specific contexts of human activities. Personas [32] are a means of representing potential users and contexts of use in the development process. They may help designers focus on the concrete end-users in the process, but only represent a small part of the reality. Activity analysis can offer a deep understanding of user needs and the processes and context of their activities, but it is costly and time-consuming, especially for high-expertise activities, which may be very difficult to understand and describe fully. User-centred methodologies [31], with iterative usability testing is a way to check the relevance of the concepts or prototypes. It is also very expensive, as it requires several iterations and the development of several functional prototypes, although it gives strong insights for software development. Finally, participative design [40] is a way to involve users in the development process, putting the users in a decision role. It gives a lot of guarantees about the relevance of the product and greatly favours its acceptability. But it requires the availability of end-users in the framework of an institutional context, allowing them to assume a decision role. Furthermore, those methods are often used after the definition of the project, and the design of new software or environments are thus not strongly anchored in a need analysis.

The approach we propose in this chapter is a mixture of these methods. We extend them thanks to a strong anchoring in the professional domain and in the market. Collaborating closely with the architectural domain allows our development team to anticipate users' needs and to design adapted responses to them.

The way we define needs is based on a multidisciplinary approach. The competencies involved can be defined along three axes, working together on development projects to enrich their mutual reflections:

- The domain axis, composed of architects, building designers or structure engineers. This axis holds all the technical knowledge of the architecture domain. They know the practices, the tools and the issues related to the domain. But they do not constitute a group of users, being inserted in a research context and not in a professional or industrial context.

- The usage axis, with ergonomists and work psychologists. This axis has the responsibility to lead the participative design process, to gather information about real activities of architects and to manage the ergonomics aspects of the user interfaces

- The development axis, with computer scientists and computer graphics designers. This axis has the responsibility to design and develop applications based on the identified needs and requirements from the other actors.

These three axes collaborate on application development in the following way (figure 1) : the domain axis and the usage axis work together to feed the development axis, which will design the application. This application (being in a concept stage, a prototype or a final product) is tested with real users. This leads to a new development cycle which in turn is tested, forming an iterative process. 


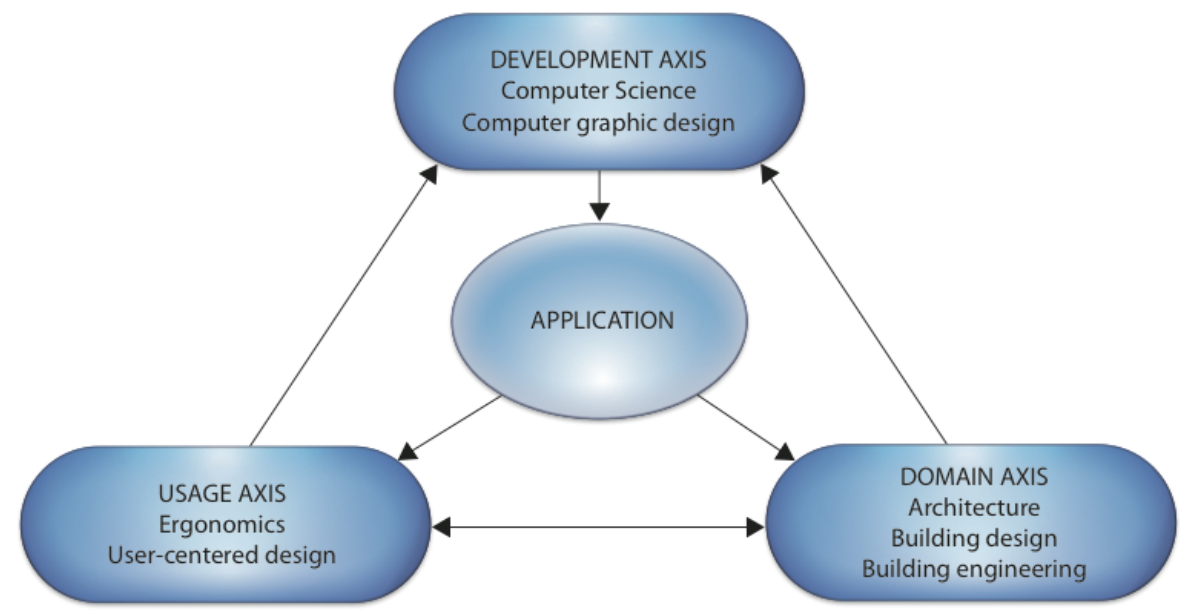

Fig. 1. Multidisciplinary methodology

Concretely, this means several steps and methods for each research or development project:

- Formation of a user group. This group will follow the project from its beginning to its end. These users are architects in professional situations. They are selected for the diversity of their practices: size of their office, type of architecture, background, age, etc. They meet together on a regular basis, to be informed of the development of the project and to react to some concepts and ideas. Furthermore, this group will provide users for interviews, observations and application testing.

- Project meetings. Each time decisions have to be made, members of the three axes are involved. The development axis will handle the technical constraints; the usage axis will deal with the constraints linked to usability and the context of use ; and the profession axis will bring issues about the meaning and the utility of the concepts discussed. This separation of concerns supports an integrated reflection that will lead to solutions that take into account the functional, technical and cultural points of view on the development.

- Focus groups. The user group is meets regularly to respond to specific questions on which the project team has no definitive answer. Concepts and ideas from the project team will also be validated with focus groups.

- Evaluations. At key moments, the prototypes will be evaluated. These tests bring the strongest feedback on the efficiency of the solution. These evaluations take place in a setting as natural as possible - from realistic tasks to the placement in the user's office.

- Industrial partnership. In addition, all the projects are followed by industrial partners. They are informed of the developments, participate in key meetings, and ensure the feasibility of the development.

- Many demonstrations and close relationships with the industrial actors. Apart from the user group and the industrial partners, the lab tries to communicate widely with the domain actors, by making regular prototype demonstrations. This helps to have informal feedback on the development, anchored in very diverse realities. This also helps to anticipate future needs and identify related domains.

This approach is interesting because it guarantees the involvement of the users (or at least some domain experts) at each step of the development process, starting with the definition of global objectives. It allows a deep understanding of complex high-level activities, as creative design and architecture, and the co-construction and exploitation of knowledge about these activities. The close cooperation between the three axes and informal communication enable a strong reactivity at each step of the development. This reactivity allows the development team to discuss each aspect of the application throughout as a negotiation between the three axes, in real-time. The whole approach is designed to guarantee the adjustment of our solutions to either the cognitive processes of potential single users, but also to the needs of the market. The everyday focus on the usefulness of the application and its usability, strongly anchored to the domain, may lead to a great enhancement of the user acceptance. 


\section{Context and needs}

\subsection{Architectural Design}

An architectural project is a long process characterized by several steps, several actors and several tools. An architect's work begins by determining the client's needs and ends with the construction or renovation of a building. The architectural project, which may last several weeks or several years, currently suffers from a fragmented approach. This breakdown stems from different legal responsibilities at the various phases. It results in various sorts of representations that may or may not make use of computer tools (Figure 2).

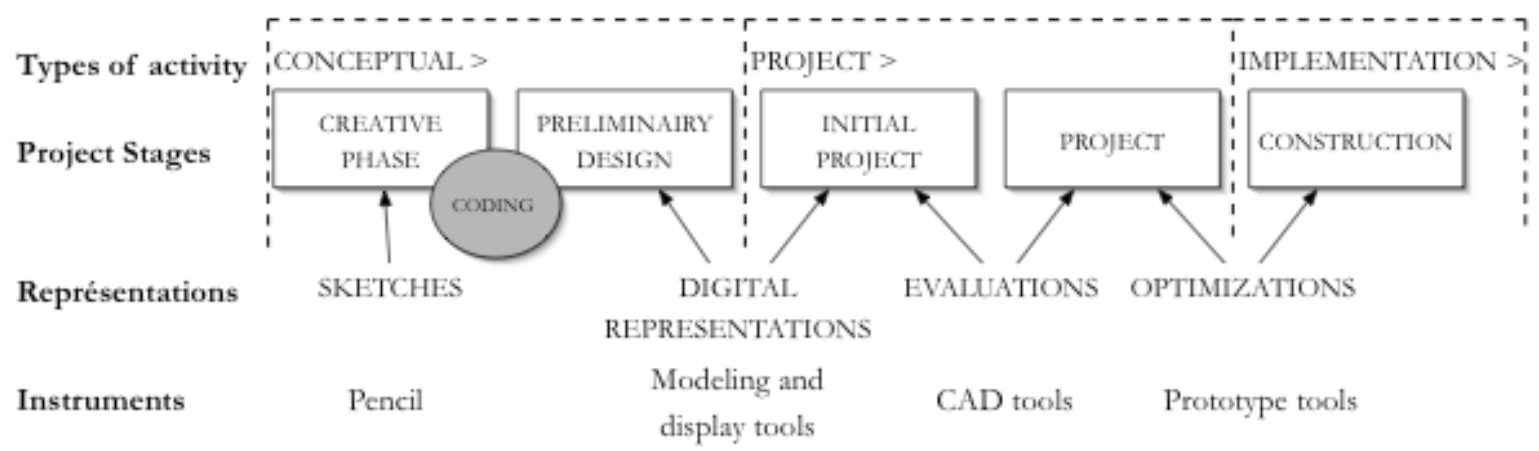

\section{Fig. 2. Phases of an architectural project}

The first phase is the creative process during which the architect generates ideas and gradually recognizes constraints and criteria involved in the project undertaken [21]. While the subsequent phases of the project are largely supported by information technology, this initial phase is essentially a pencil and paper exercise. After making sketches, the architect has to go through the fastidious step of encoding the sketches on the computer, which is necessary to study the feasibility of the contemplated solution. Next, there are a number of production phases that precisely define all aspects of the building. These phases include comprehensive geometric resolution and complete parameterization of the architecture in order to draw up plans that are communicated to the various parties involved in the project. Finally, in the construction phase, the architect supervises the project. The distinction between creative design and engineering activities is relevant because different types of instruments are used, different cognitive processes brought into use and different types of representations involved.

All these activities are becoming progressively more collective. The architect doesn't own all the necessary technical knowledge, and several experts (structure, acoustics, energy needs, etc.) have to intervene based on the plans. The different collaborators sometimes work together from the beginning of the design. The conceptual design becomes collective. Moreover, those collaborators are usually working in different places, sometimes quite distant from each other. In these cases, meetings can be very expensive and time-consuming.

In this context, and based on our observations and knowledge of the trade, we can identify two different technological gaps, i.e. two activities that are not currently supported by information technologies : real-time collaboration at a distance, and the preliminary conceptual sketch-based design stage.

Many CAD tools already allow ideas to be designed and directly manipulated digitally. However these tools fail to help the designer in the initial design phases, i.e. when the broad outlines of the project must be defined and the crucial option chosen [2, 12, 42]. One of the explanation for this failure relates to the user interface : these tools require painstaking coding of precise data which is only possible once the project has largely been defined [22]. Moreover these systems place users in a circumscribed space. Their movements are reduced to mouse shifts and clicks, and their sensory interactions are limited to passive visual and auditory simulation. A second reason is related to the sketches widely used at the start of the design process. The sketch is used as a graphic simulation space [18]: basic elements of the project set down in the earliest drawings are progressively transformed until a definitive resolution is achieved. Each sketch represents an intermediate step from the first rough sketch to the definitive design solution.

In the same way, there are several tools supporting remote communication, becoming over time more powerful, less expensive and easier to handle. For example, e-mail is a great asynchronous communication tool. 
Online exchange databases allow the easy sharing of large files for cooperation. Video-conferencing is a powerful tool for real-time collaborative discussion. But these tools fail to really support collaboration during the creative design process in architecture : there is a need to share in real-time the products of the early design, the sketches.

In the following sections we describe these two issues more deeply.

\subsection{Sketch-based preliminary design}

The sketching phase, one of the first steps in an architectural project, represents an important part of the process. It is usually described as a trial-and-error process. Errors are very common and they are quite cheap to recover from. The sketching phase, in case of huge errors, allows the designer to start the design again "from scratch" and change the concepts. Errors can also be very productive, but, as the process goes further, remaining errors become considerably more expensive and their recovery becomes more difficult. In the production phases of design, it is not possible to change concepts but only to correct them (and sometimes not completely). This emphasizes the need to assist designers to detect and correct their errors during the sketching phase, in order to detect them before it is too late (when the price of design is already too expensive).

In current design practice, preliminary drawings are essentially produced on paper before being converted into a representation in a Computer Aided Design System (CAD). Why do designers still prefer hand-drawn sketches to computer-assisted design tools at the beginning of the design process? According to McCall, Vlahos \& Zabel [28], there are three reasons : first of all, the pen and paper technique leads to abstraction and ambiguity which suit the undeveloped sketch stage. Digital pictures, hard edged, are judged more finite and less creative than traditional sketches, fuzzy and hand-drawn. Designers need freedom, speed, ambiguity, vagueness to quickly design objects they have in mind [1]. Secondly, it is a non-destructive process — successive drawings are progressively transformed until the final solution is reached - whereas CAD tools are used to produce objects that can be manipulated (modification, destruction, etc). Thirdly, sketching produces a wide collection of interrelated drawings, while CAD systems construct a single model isolated from the global process. Moreover, the sketch is not simply an externalisation of a supposed designer's mental image; it is also a heuristic field of exploration in which the designer discovers new interpretations of his own drawing, opening up a path to new perspectives for resolution $[11,23]$.

Whereas digital representations are by nature unequivocal, the sketch is sufficiently equivocal to produce unanticipated revelations [44]. Sketches, externalizations of the designer's thoughts, allow the designer to represent intermediate states of the architectural object. In addition to this function of presenting and saving information, sketches play a role as mediator between the designer and his solution. They are cognitive artifacts in Norman's [29] sense of the term, enabling the individual to expand his or her cognitive capacities. They are rich media for creativity: the ambiguity of the drawing enables new ideas to emerge when old sketches are getting a second look. The architect voluntarily produces imprecise sketches in order to avoid narrowing too rapidly to a single solution, and to maintain a certain freedom in case of unexpected contingencies during the process.

Scientific studies of the sketch identify the operations that emerge from producing a series of sketches, in particular the lateral transformations, where the movement involves passing from one idea to another slightly different idea, as well as vertical transformations where the one idea moves to another more detailed version of the same idea [10]. Goel also shows that through their syntactic and semantic density, as well as their ambiguity, freehand sketches play an important role in exploratory activity. He suggests that the properties of freehand drawing facilitate lateral transformations and prevent early fixations.

The conceptual sketch has different graphical characteristics than digital representations produced by CAD software [21, 23]. There is not much variation in the lines used for the sketch (types of dotted lines, colors, thicknesses, etc.) and the lines are very imprecise. The drawing is incomplete and shapes often begin to emerge from an accumulation of lines, sometimes redundant. A number of solutions may coexist on the same sketch. This type of drawing is extremely personal, which limits understanding by another person. On the contrary, the clean plans produced using CAD tools are complete and precise. They provide a single and unique representation of the architectural object. A variety of highly codified lines are used. There is no difficulty communicating these plans to someone else and they are produced with exactly this objective in mind. 
Therefore, a system that aims to support the graphic conceptual activity should satisfy the following requirements :

- Allow the natural drawings of sketches, that are the primary ways of thinking.

- Do not interrupt the designer's flow of thinking.

- Allow multiple representations.

- Allow incomplete description of the architectural object,

- Allow the use of imprecise descriptions of the architectural object.

- Help to broaden the point of view of the designer to help her produce transformations.

\subsection{Distant collaborative design}

In a wide range of types of activity, collaboration has intensified, notably in the design domain. Collective work is increasingly organized simultaneously, rather than sequentially as it used to be in the past. Moreover, design teams are often spatially distributed, and the need for distant real-time interaction is consequently emerging. A lot of effective systems are available for sharing information, but most of them are asynchronous (e.g. databases, email, etc.) or allow only partial interaction (e.g. phone) .

Therefore, a tool to support distant sharing must respond to the following needs:

- Provide a natural pen-based environment, without wires, mouse or keyboard to support normal drawing. Moreover, working together on a shared space generates the need for a wide space, to express various and different points of view on a same subject. This environment must therefore have a large size (comparable to a drawing table), but also the right level of resolution in order to provide the requested drawing precision.

- Support free-hand sketches, drawn from distant locations on a shared work space in real-time. The method of sketching must be as close as possible to the "classic" face-to-face drawing session, with a shared sheet of paper. Designers thus can express more ideas, concepts and alternatives than in long distance asynchronous collaborations, which inevitably and obviously provoke delays, misunderstanding, hazardous interpretations, loss of documents and coordination problems [13].

- Provide an awareness of the situation (who is around and what is happening) by supporting, in real-time, a global and multimodal overview of the context.

- Support the integration of additional reference material (notes, plans, manuals, etc.) generally required for collaborative work.

\subsection{Why mixed reality should be a good way of responding to these needs}

Virtual reality is a promising way to respond to the challenges of changes linked to collaborative design and sketch-based design in organizations and processes. The aim of mixed reality is to enhance the individual capabilities (mainly in terms of information perception) without cutting him off from the real-world, i.e. breaking the frontier between the real and the virtual while helping the user to complete a task [8]. Yet, that is the main problem identified in the needs discussed above: designers, in order to benefit from virtual tools, have to deviate from their natural way of working. Evaluating the performance of a building implies switching from creative sketches to formal CAD drawings, and communicating at a distance implies switching from rich multimodal communication in meetings to single channel or asynchronous communication.

The LUCID-ULg Lab proposes a system for sketch-based multimodal interaction based on the paradigm of the invisible computer [30]. Instead of requiring designers to change their way of conceiving a design, we propose to support one of the most usual ways of collaborating: free-hand sketching, which plays an important role, especially in the initial stages of design.

As we show below, our response involves a system where there is a mixture of realities, allowing for interactions between the real environment (the table, the pencil and the colleagues) and the virtual environment (the virtual shared sketches, the interpretation and evaluation of the building). The frontier between reality and virtuality is particularly obvious in the series of graphic representations produced by the architect. Our solution as part of the "invisible technology" movement is to emphasize seamlessness between real and virtual worlds [30,34]. When applied to architecture, the concepts of simplicity (complexity should be a characteristic of the task not the tool), of versatility (the apparatus should be designed to encourage new and creative interactions) 
and of pleasure (the tools should be pleasant and amusing) take on a particular significance. We have attempted to infuse these concepts into the prototype that we are presenting here.

\section{Technological solutions}

\subsection{Introduction}

This section describes the hardware and software implementation we have built to produce an immersive environment for architect and designers. This environment consists of a virtual desktop on which two different systems are running. There were some general requirements that we constantly kept in mind, in order to guide our technological choices, including:

- Follow the 'disappearing computer' paradigm. It means the software we need should reproduce the usual environment and working habits of the user as closely as possible.

- Provide a large working area. Usually, architects are used to working on large drawing boards (typically, around 1.5 large x $0.6 \mathrm{~m}$ depth).

- Pen-based interaction. Architects and designers are very much used to drawing (while most mechanical engineers, for instance, have much less experience of drawing).

- Augmented sheets of paper. It imitates traditional paper (layers with transparency support, graph paper), with augmented capabilities (very large size, zoom, scale and rotation functions, and customable transparency).

- Allow traditional interaction. Real drawing tools, like rulers, will be allowed. Also, the digital table can be used as a real table (on which to place tools, real paper documents or a coffee mug).

- The compatibility with real paper documents should be supported (typically by importing them as JPEG in our stack of virtual sheets).

- Provide a good level of resolution for the drawing.

\subsection{The Virtual Desktop}

The Virtual Desktop (figure 3) is the hardware part of our mixed-reality immersive environment. It is made up of three main core elements (the computer, the digital table and projectors encased in a suspended ceiling), and a few additional extensions (a video-camera for gesture recognition and a videoconference system for collaboration).

The computer we have chosen offers two DVI (video) outputs, in order to connect to the two parallel projectors. It is linked to the table through a USB connection. It runs the dedicated applications, but is also responsible for managing calibration (synchronization between the digital table coordinates and 'screen' coordinates). The digital table offers a comfortable drawing area, with its A0 dimension. The suspended ceiling is a work of joinery, built in wood, and specifically designed for our research needs. It stores the two projectors, and possibly the computer and the video-camera. 


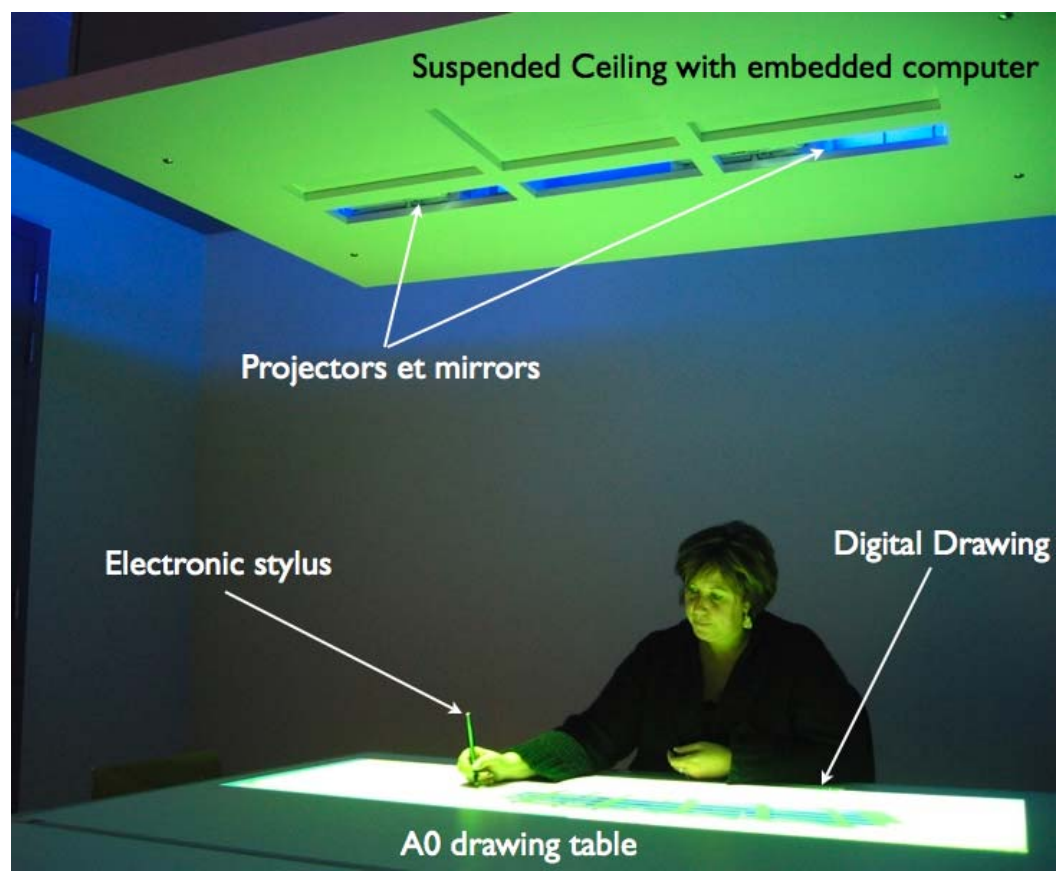

Fig. 3 Virtual Desktop.

The disposition of the table and the projectors have required some adjustment. The first idea was to project the working space from below a table. Tests have showed that this projector encumbrance under the table made the designer's leg position uncomfortable (which doesn't suit long working sessions), did not respect the disappearing computer criterion and constrained the designer's freedom of movement. Inclined worktables are not suitable for design because the designer usually needs to work with a lot of paper sheets. It also becomes uncomfortable to work on such a table after several working hours. So we preferred a simple horizontal whiteboard with a projection from above.

In the first release of the Virtual Desktop, a projector was placed in a suspended ceiling with a classical mirror. To obtain a larger work surface and to increase the precision (the target was $4 \mathrm{pts} / \mathrm{mm} 2$ ), a second projector was later added to the Virtual Desktop. This change required the resolution of a technical challenge: to manage one screen with two projectors and obtain precise lines efficiently.

In order to clear shadows arising from projection from the ceiling, projectors and mirror were installed to make the projection inclined, so that the user sits out of the projection beam (figure 4).

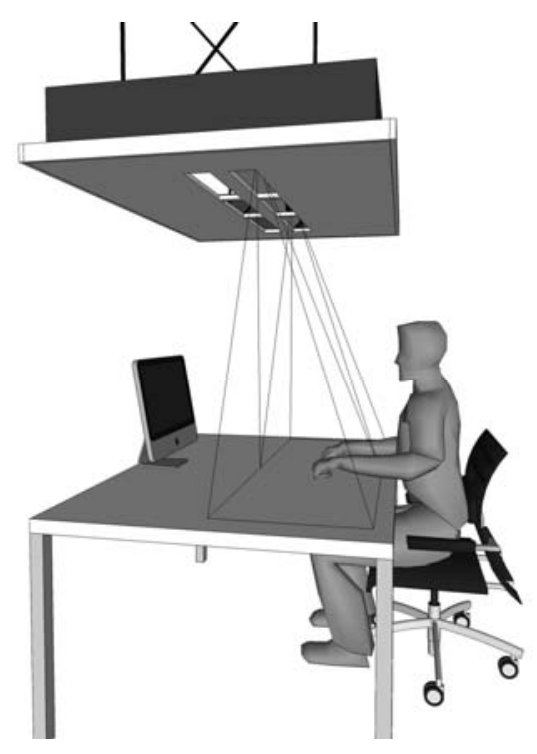

Fig. 4. User's position related to projection cone 
The first release of the desktop was realised with a pen-positioning system based on infrared and ultrasound triangulation. Two problems lead us to replace it. On the one hand, the pointer, bigger than usual drawing tools, is quite difficult to handle and adds shadow areas on the drawing. On the other hand, the system has a centimetric precision which is much too low for architectural sketching.

In the second release, a selenoid digital pen has been chosen with several benefits: it has much finer precision and does not add large shadows areas; it doesn't need a power source which makes it lighter and less bulky; it is more stable and easier to handle; it is also more accurate than the infrared pen. It perfectly matches the characteristics of a usual pen.

Projection from above allows the coexistence of real and virtual drawings, and a simple integration of penand paper drawings in the system : the user can highlight real documents with the electronic pen, retracing the drawing to import it in the virtual world.

If we compare our Virtual Desktop with the Microsoft Surface table, for instance, we can easily show that the latter is not suitable for long working sessions, as the user cannot put his knees below the table. Another well known smart surface is the DiamondTouch table, produced by Circle Twelve Inc. It uses a similar projection from above like the virtual desktop, but with only a single projector, reducing the available working area. Also, these two systems primarily aim at finger manipulation, and don't offer pen support. They target more general applications, while the Virtual Desktop mainly focuses on designers’ applications.

Additional components can enhance the Virtual Desktop. A webcam on a 24 inch screen computer offers the ideal extension to support a video-conference session when remote collaboration is needed. We have also added a video-camera in the suspended ceiling : through image processing, we can evaluate gesture recognition as an extended interaction modality. Currently, we are able to transmit 'finger pointing' to the partners in a collaborative session (see [43]). In the near-future, we hope the system will react to hand movements, so that the user can organize his sheets of digital paper more naturally with simple gesture.

\subsection{EsQUIsE}

\subsubsection{Introduction}

EsQUIsE is an application dedicated to the early stages of design in architecture. The user simply draws his sketches as he would do with normal paper. This way, his creative process is not disturbed by the application, because he doesn't have to explicitly declare his intentions to the system.

EsQUIsE assists the user by providing relevant information (3D model, topological model and energetic needs) on the building being designed. The software architecture consists of three modules (figure 5): the entry module, the interpretation module, and the evaluation module (for more detail see [14, 15, 19, 20, 22]).

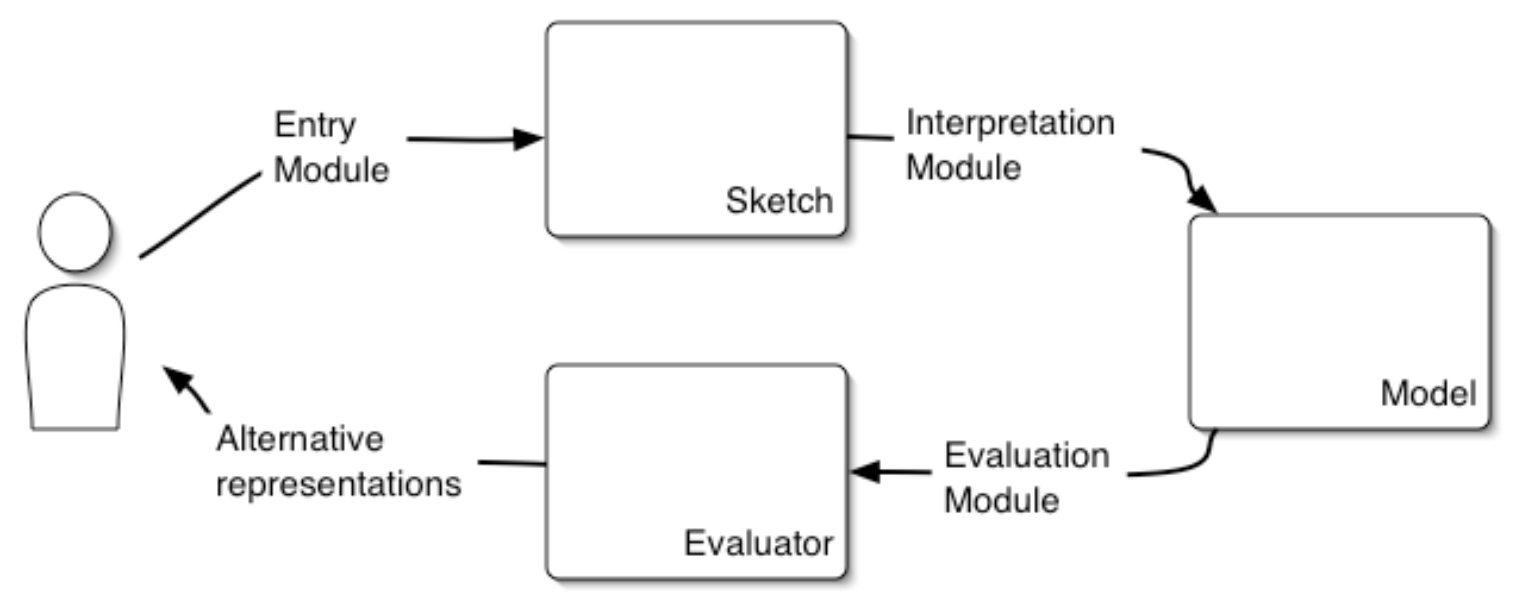

Fig. 5. EsQUIsE Architecture 


\subsubsection{The entry module}

The role of the entry module consists of analyzing sketches in order to construct a geometrical model of the drawing, i.e. the significant graphic elements and the relationships they maintain. The principal constraint on such a system is obviously the requirement that it should work in real time. Analysis therefore takes place in two phases. While the electronic stylus is being moved over the pad, the system captures the designer's movements. Then, as soon as a line is finished, the system takes advantage of the time lapse available before the start of the next line to run all the procedures to synthesize and analyze the layout. The synthesis module consists of successive filters intended to extract the essential characteristics of the lines, reducing as much as possible the amount of information to be processed while conserving as faithfully as possible the appearance of the original line. To ensure that the sketch retains its « triggering potential » this step is carried out transparently for the user, who only ever works on his or her initial drawing, unaware of any interpretation being made by the system.

Once the strokes have been individually processed, EsQUIsE captures the relationships between them by using a combined technique of segmentation (to differentiate and to compose sets of lines which « work together ») and of classification (grouping lines according to their characteristic criteria). To reach that goal, we have implemented a multi-agent system, which allows the system to consider multiple scenarios at the same time, until enough information is available for a classification decision. Beyond some particular symbols (hatching, blackening, dotted lines, underlining, etc.), the system also focus on the identification of the written captions which it identifies and situates on the sketch. The aim of the analysis of the drawing is to weave the network of relationships between the different graphic objects it contains. Relationships include, for example, inclusion, intersection, proximity and superposition of lines and contours. Because the sketch is imprecise, we have developed a «fuzzy graphics » approach that takes into account a considerable margin of error in the identification of points, lines and intersections. Outlines, for example, do not need to be fully closed-off in order to be recognized; by analyzing the proximity of the ends of the lines, EsQUIsE is able to identify an imprecise outline.

\subsubsection{The interpretation module}

The role of the interpretation module is to translate the geometrical information, produced by capturing the sketch, into a functional model of the planned architectural object. This model is generated from collected semantic properties. Lines make outlines which are translated as rooms. Other lines are recognized as letters forming parts of legends. Those are completed by implicit information known by the system. For example, the word « bath » translates on the sketch the function that the designer gives to the designated space. The outline in which this word appears becomes a function-space in the architectural model with characteristics for this room: a $24^{\circ} \mathrm{C}$ temperature, a high humidity level and an average noise level. Those parameters, given with any designer's intervention, are used by the system to fix technological choices: the lines separating the outlines are interpreted as walls separating the rooms. Those, being characterised, are able to find their own technological composition in order to modulate heating and humidity flows passing by the walls. Operating in the same way with the geometrical characteristics of the rooms (ceiling height for example) the system is able to compose in real time, a complete and cogent (coherent) model of the building being designed, from some sketches drawn by the designer. This whole process is carried out in the background, without requesting the user to declare his intention explicitly, so that he can truly focus on his creative task.

\subsubsection{The evaluation module}

This building's model may therefore be used as a source for several evaluations. The main one is the production of the 3D model through which the designer can virtually walk and that allows him to check the representation of the dimensions and spaces he thought of. EsQUIsE is also able to use this model to estimate the energy performance of the future building and so give the designer a good idea of his/her options. Simulating the solar source, knowing the desired temperatures for each room and each wall's composition, this second module may estimate the needs in heating or cooling the building.

Figure 6 shows the latest version of the software interface, which is purposely kept as simple and uncluttered as possible. 


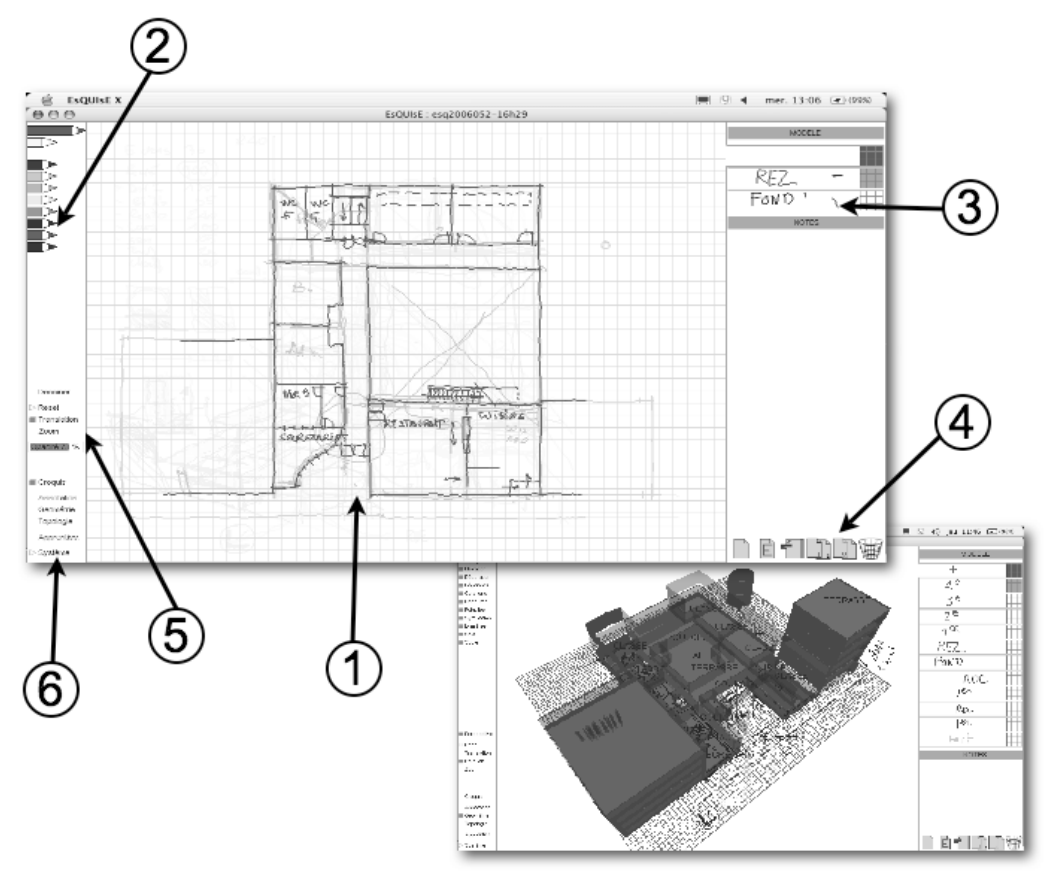

Fig. 6. EsQUIsE interface

In EsQUIsE, all the operations are driven by a single stylus. A large area (1) is available for drawing virtual sketches using a palette of virtual pencils and erasers (2). Each sheet of tracing paper is indicated by a tab (3). The user can name these tabs. He can also arrange them, simply dragging and dropping virtual sheets of tracing paper to superpose them by within the model. The user clicks on editing icons (4) to create, delete or duplicate each sheet of tracing paper. Tracing sheets are handled (rotation, shifting places and zoom) using tools available in the image handling area (5) and parameters can be set for the level of transparency of the tracing paper. The option area (6) enables the user to pass from one mode to another (sketch mode, 3D mode, other digital architectural evaluators, preferences, etc.). The building designer can sketch freely on the virtual tracing paper with digital pens that can draw in different colors. EsQUIsE only interprets black lines in making a model of the building. Other colors are used for idea sketches and annotations. The tracing sheets are semi-transparent and can easily be arranged in relation to one another.

\subsection{SketSha}

The SkeSha software offers a shared drawing environment. It allows several remote workstations to be connected to the same drawing space, in order to support collaborative design. The application captures the strokes that compose the sketch, shares them between the different distant locations (through a classic internet connection) and transmits the whole information in real-time on the active boards through video-data projectors. Some CAD facilities have also been introduced in the prototype, such as the possibility to manage different layers and sheets of virtual paper, to delete or reproduce them, and to manage their transparency. The software also allows the import of plans and images. Pointing, annotating and drawing are possible thanks to the electronic pen.

The system is completed by a 24 inch display and an integrated camera, that allow the participants to see and talk to each other in an almost 1/1 scale during a real-time conference. This integrated camera is in fact a very simple way to avoid the deviation of the look when talking to the interlocutor(s) (see figure 7 for the whole environment). Moreover, a gesture recognition module based on computer vision is currently being developed. In the near future, this system will be able to capture gestures and finger-pointing on a Virtual Desk, and send the information to other connected desks, which then will display a "hand avatar" of the distant partner. 


\section{Fig. 7. Distributed Collaborative Design Studio}

The application structure can be seen as two separate modules: the graphical interface designed to be as natural and friendly as possible, and an efficient network layer.

The graphical interface is composed of 4 elements:

- The main drawing area, designed to cover the whole surface of the virtual desktop.

- The pen toolbar, where the user can select a pen (with a specific color or thickness) or the eraser.

- The 'pages' toolbar where the user can create new sheets of paper, duplicate and delete some, or reorder them as he wishes in a layered stack.

- The navigation widget, dedicated to our pen-based environment. Because of the large working area, the widget is called with a simple 'press and wait' operation with the pen. Once it is under the pen, the user can control the view with the classical 'translate', 'rotate' and 'zoom' operation.

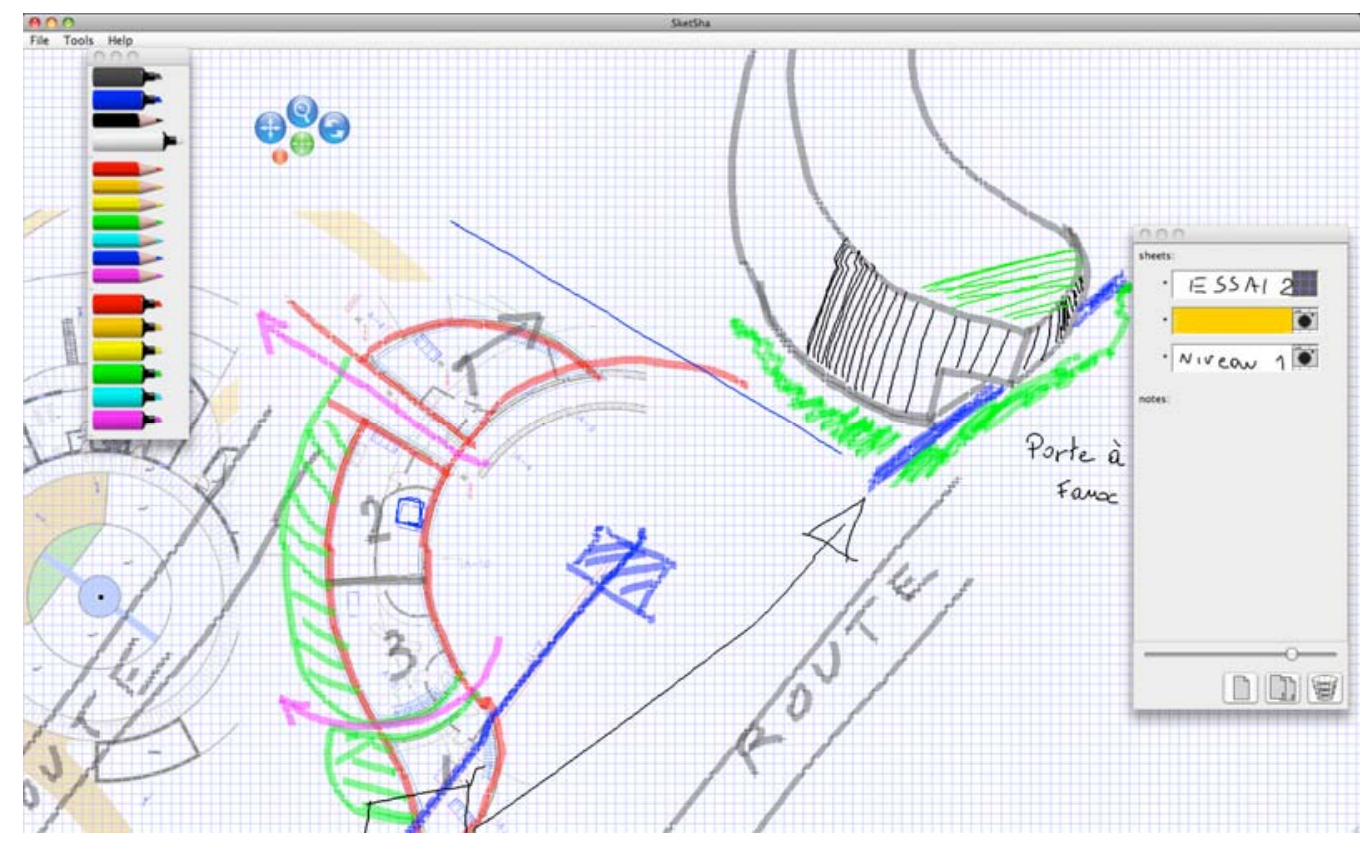

Fig. 8. SketSha interface 
The network layer is responsible for distributing the different actions from/to all users. It has been designed with the following requirements in mind:

- The project view must be exactly the same for all the users (if the screens have different sizes, the centre point of the view is shared). If a user changes the sheet order or changes the current active sheet, it will impact all the users.

- The drawing must be in real-time: if the network fails to provide proper bandwidth, a stroke that is being drawn should not be affected (the local rendering must happen as fast as possible whatever the network).

- We want the system to support multiple clients. Tests have been successfully conducted up to 5 users in the same session.

- We want an optimized use of the network capabilities. If multiple view changes are queued somewhere in the network flow, they can be reduced to a single one. This process is called 'coalescence'.

There is an apparent paradox between the real-time requirement (" process my data first »), and the sharing of the same view (" process everybody's data in the same order for every client »). To solve this problem, we have chosen to encode the multiple actions as messages transmitted over the network to a central server (one client is responsible for acting as the server). The server puts all these messages in a single queue to order them, then redispatches them in that order to all the clients. These messages have properties to describe their 'coalescing' behaviour or 'real-time' behaviour. Thanks to these qualifications, we can properly process the messages to reach the given goals.

\section{Evaluations}

The Virtual Desktop and the two software prototypes described above have been tested many times, in a wide range of situations:

- in long individual work sessions with students in architecture course [7, 35, 36, 37, 38, 39] ;

- in long individual work session with architects [6, 24, 25, 26, 27];

- in collective sessions with students in architecture $[4,5]$;

- in a pedagogical long-term distant collaborative work setting $[9,16,17]$;

- in collective work sessions with professionals (engineers and architects) and

- in many short demonstrations and trials with professionals from various industries.

Most of these evaluations and tests have been videotaped (fig 4) and analyzed by ergonomists. These studies had different goals from each other, and therefore the observations were based on different evaluation criteria. But they shared some common topics:

- the usability of the system and the way they're handled,

- the expressive power of sketches to support the activities,

- the immersive aspect of the virtual desktop,

- the influence of the technology on the quality of the design process.

In this section we synthesize the main results of these studies.

\subsection{Usability}

In all the tests we've made, the most interesting result may be the simplicity of the systems: it takes very little time for any user to completely master them (less than half an hour for EsQUIsE and less than 10 minutes for SketSha). Furthermore, in long sessions, the systems seem to disappear from the consciousness of the users.

If we investigate each functions separately, we can draw the following conclusions:

- The navigation widget is a very powerful tool. It is perfectly assimilated by the users, and allows flexible manipulation. In comparison with EsQUIsE menu setting, the manipulations done with the widget are more accurate, more precise and above all much faster.

- The principle of overlays is very efficient: icons are well understood, the transparency setting is often used and editing functions seems to appear quite useful. Therefore, it allows the same kind of use as conventional tracing paper, being augmented by the ease of management (calibration from one layer to another is 
automatic) and the editing functions. But, it has a limitation: the layers cannot be moved one in relation with each other in the horizontal plane. This function could be useful in order to change the point of view of the designer and to be flexible.

- The pen-based interface is easy to handle. But the current technology raise quite an annoying problem: only a single stylus can be used at a time on a single table. Therefore real-time collaboration is much more efficient in distant situations than in co-present situations.

They also show the richness of the interactions supported by our collaboration system. They demonstrate its ability to support distant collaboration as if the users were copresent. The long term sessions have also shown that the system was an efficient cooperation tool for concrete projects.

\subsection{Sketches}

We have shown [37] that the sketches on EsQUIsE, although being less precise than paper sketches, contain the same quantity of information than the latter. But we also have shown previously [39] that using the system implies an interesting diversification of sketches: in EsQUIsE, the user separates the "rough" sketches from the "clean" sketches in different virtual sheets. This differs from traditional pen and paper drawings, where a single sheet may contain both rough and clean sketches. We explain this difference with the modalities of construction of the 3D self-generated model. It illustrates the distinction between two types of graphic output in the creative stage of conceptual research for an architectural project (figure 9):

- Simulation sketch. An architect must have the necessary conceptual tools at his disposition to allow for a flexible and unlimited search for solutions [18]. Rough drafts spontaneously produced by building designers fulfil this function. These drawings are characterized by a high level of ambiguity and personalization, as well as by a proliferation of graphic solutions to architectural problems. This is what makes it difficult, if not impossible, for these sketches to be understood and used by another person

- Synthetic sketch. This sketch results from finishing activities. A unique solution is represented and emerges from a choice of structuring lines selected from among the large number of lines present in the simulation sketches. The drawing is less ambiguous and more conventional than the simulation sketches. The synthetic sketch can be largely interpreted by another architect.

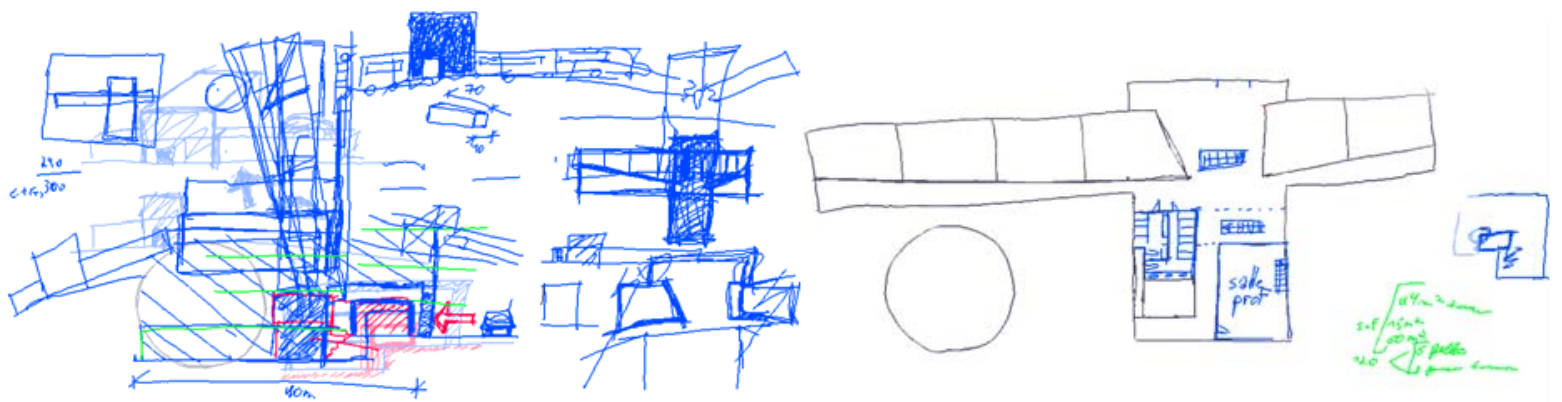

Fig. 9. Simulation sketch (left) and synthetic sketch (right) for the same building design.

EsQUIsE and the Virtual Desktop are therefore capable of hosting the multiple sorts of drawings that occur in the preliminary phase of architectural design, all being involved in the man-machine dialogue. But this also shows that these two kinds of drawings are voluntarily separated in EsQUIsE. Therefore, we think that the model of "comprehensive interpretation" is a bit of an illusion. If the objective is to interpret conceptual sketches, we think that the simulation sketch is and should remain personal. Its very ambiguous and idiosyncratic nature lends itself to a difficult computer (or even human) interpretation. On the other hand, the finished drawing or synthetic drawing, can be interpreted and contains all of the information necessary to make a model of the building. 


\subsection{Immersion}

An essential point of interest of the virtual desktop, in comparison with classical pen-and-paper activities, lies in the methods of sketch exploration. The exploration of drawings is indeed an important element of the architectural conception. Having new points of view on the drawing provides the emergence of unexpected discoveries [41] and of what Goel [10] calls " lateral transformations», i.e. the movement from an idea to another.

During the design the sketches are explored by the architects. There are movements on the paper sheet, allowing explorations for the formalization and the expression of the non-graphic ideas and expression of some intentions, especially in terms of circulation of people and space dynamics. In classical pen-and-paper activities, the sketches are explored with the pen: some elements are pointed to bring them to the fore in the architect's reasoning and the pen is often in movement to explore the circulation in space or some other dynamic concepts.

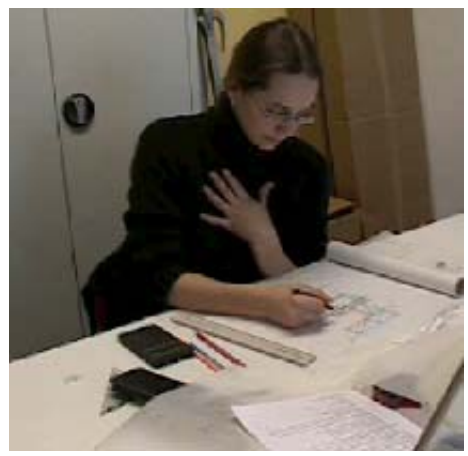

Fig. 10. Exploration by pointing on paper/pen

In virtual desktop activity, even though these methods of exploration are present, we also notice a bigger physical investment in the drawing: several times, the whole hand allows the expression of a movement and the use of the two hands allows a space delimitation or a intention expression related to a surface (see figure 10). This can be explained by the bigger size of the drawing on the virtual desktop, but also by the immersive aspect of the system. In fact, several factors facilitate the immersion in the virtual desktop:

- The luminosity of the work surface contrasts with the darker environment of the room.

- The environment's “embodying aspect”, places the designer between the table and the suspended ceiling, in a delimited space and explicitly dedicated to the design.

- The presence of a large and coloured dynamic 3D-model, contributes to work space specialization and keeps the user's attention on the system.
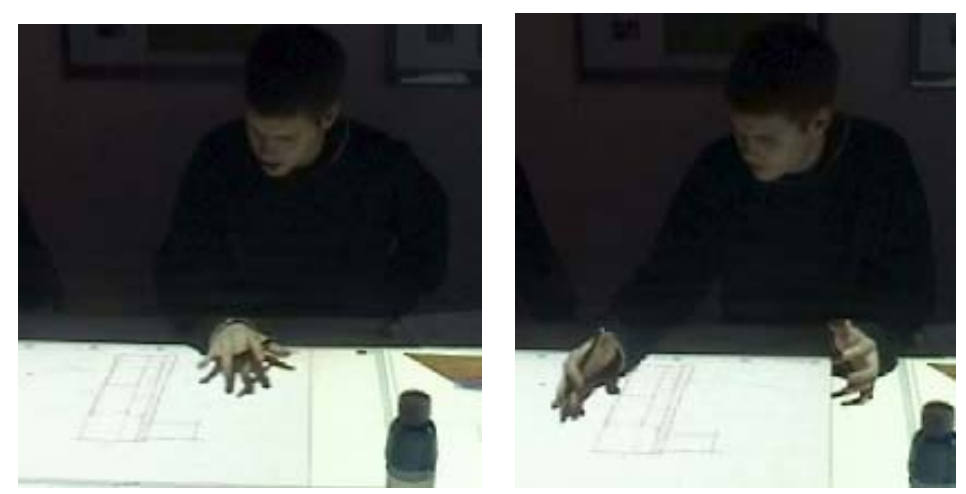

Fig. 11. : Exploration by hand movement and space delimitation with two hands in the Virtual Desktop

\subsection{Design process}

Long-term evaluations of EsQUIsE have shown the following results (for more details, see [39]): 
- The 3D model is a good tool for evaluating building volumes, used at some key moments to check the effectiveness and the aesthetics of the design. But it doesn't allow the direct expression of three dimensional ideas, which is an important function of the volumetric drawings (e.g. perspective drawings). Furthermore, the 3D model is used mainly by students. Professional architects seem to be more confident in the volumetric aspects of their design, and thus don't need to evaluate and continually check their building's volume.

- The 3D model and the interaction modalities of EsQUIsE confer a structuring impact on the design process. The designer's way of drawing is directed towards the definition of an efficient model. It seems to have an influence on design strategies, on the way floors are designed one related to each other, for instance.

- This structuring aspect leads to a spontaneous differentiation of sketches in the system, as explained earlier. The users seem to differentiate their own personal drawings (simulation drawings) and the drawings they keep for communicating in a less ambiguous way with the computer (synthetic drawings), as they would do with other human partners. They use different virtual sheets for these different kinds of drawings. We interpret this observation as an intention to hide a part of their design from the system's interpretation. They want to keep their simulation drawings uninterpreted. New tools should be implemented to allow the coexistence of the different drawings, and to communicate which sketches have to be interpreted.

About SketSha, the system seems to enhance the quality of collaboration. But it raises several issues:

- Design of work space. In order to enhance the collaboration, a reflection on the workspace has to be driven. In particular, we shall investigate the usefulness of implementing private design workspaces in addition to the shared space. The orientation of the drawings may also be studied : the distant table could share the same orientation, or the drawing can be inverted to simulate a face-to-face arrangement.

- Integration in industrial practice. As the system seems to be really useful in experimental settings or for pedagogical purposes, how can it impact professional industrial practices? The first comments from our industrial partners indicate that the system won't replace all needs for real meetings, but can support day-today collaboration, and indeed enhance trust between distant partners and provide organizational communication.

- Ownership of information. In a design project, the different partners may have different responsibilities. If the whole information is shared, it may cause trouble for keeping track of the decisions, and thus for keeping track of responsibilities. Modalities of information tracking and labeling should be designed.

- Ways of collaborating. Will the system radically change the way designers collaborate? Or will it need the definition of particular roles? Future long-term studies will inform us about the way people organize their work according to the possibilities of the system.

- Additional modules. SketSha is designed so that external modules can easily be plugged in. We foresee many possible developments: automated collaboration analysis, or domain-specific helpers, for instance. The integration of such additional tools, and their impact on the collaboration has still to be studied.

\section{Characterisation of these mixed reality systems}

The whole system consists of a particular way of mixing real and virtual. Dubois [8] describes a taxonomy of mixed reality environment, depending on two main criteria: the nature of the object being augmented (Augmented Reality and the Augmented Virtuality) and the type of augmentation (execution of a task or helps for evaluation).

The main issue here is to define what is the task and what is the object. The Virtual Desktop, in this taxonomy, can be considered as an augmented Virtuality execution device; the target of the activity is a virtual sketch, that is augmented by natural pen-based interaction. Therefore it aims at giving normal ways of interacting with the computer, i.e. natural ways to draw the digital sketch being processed. It helps the drawing and therefore the execution of a task. It also allows a quick integration of real drawings in a virtual world, by highlighting real pen and paper drawings in the virtual desktop with the electronic pen.

The EsQUIsE system can be considered as Augmented Reality Evaluation software. It gives calculation and information on a sketch. The object of the activity is the graphical description of the building, or even the building itself. This sketch is real, although it has been drawn on the Virtual Desktop : it is more than a simple image because it contains the graphical, topological and technical descriptions of the building, but also the history of the design process and thus offers a reflexive aspect. As we shown above, the drawing produced with the system shares the most common characteristics with pen and paper sketches. The key issue is that the object of the activity is the activity itself (the reflexive conversation between an architect and his drawings). EsQUIsE 
provides digital alternative representations of the building, in order to help the designer to evaluate the performance of his/her process and the solution being designed. This has been previously been referred as the Augmented Sketch [21], which offers assistance in the heart of the design process.

According to the same taxonomy, SketSha could be considered as Augmented Reality Task Execution software. The collaboration situation is real, based on speech, gesture and sketches. The environment helps designers to execute collaborative tasks at a distance, providing them with rich multimodal and creative interaction. This can place them in a situation close to copresent collaboration.

The whole system proposed here is designed to give a smooth integration between reality and virtuality. The drawing, although virtual, is based on a real activity that is important in the design process. The movement realvirtual-real is facilitated by several augmentations: pen-based interaction constitutes a natural way to communicate with a system, the multimodality supports rich real meetings, and the software components gives external representations to carry potential insight for the design. Therefore, the whole system is a mixed augmentation (augmenting real and virtual parts of the activity) which supports execution of the drawing via a natural interface, and the evaluation of the solution and the design.

\section{Discussions}

The mixed reality environment we designed is used for two purposes, responding to two metaphors :

- The first application (EsQUIsE) considers the virtual desktop as a metaphor for the traditional architect's drawing table. Recognizing the importance of sketches for preliminary design, while identifying the limits of existing computer systems in this context, we have designed an environment where the architect is able to draw freehandedly while the system interprets the sketches and provides him with feedback (a 3D model of the building and an assessment of its energy needs). In this 'augmented sketch' environment, the architect is also able to integrate its other productions (paper sketches and digital plans) through different importing and processing modules.

- The second application (SketSha) uses the virtual desktop as a remote meeting table, allowing collaborative design at a distance. Indeed, an increasingly number of teams must collaborate remotely while existing CAD tools mostly only allow for partial and asynchronous interaction. This setup is a combination of a virtual desktop, a standard video conference system, and networked, real-time, collaborative drawing software. The solution as a whole is multimodal in essence (gestures, speech, drawing, etc.) supporting remote collaborative design.

Thanks to their transparent interfaces, these two settings are designed to soften the link between real-world and virtual-world. The interaction modalities with the virtual sketches is comparable to the drawing modalities in a normal pen and paper environment. The intent is to support natural pen-based interaction while augmenting the drawing (by its interpretation for the first case and by its distant sharing in the second case). The idea is for the device to disappear from the consciousness of its user in order to let him focus on his actual task (i.e. designing). Mixed reality is therefore a powerful way to create complex environments, in which the user feels "at home".

The several evaluations presented in this chapter, made in different contexts and with different kind of users, have shown the relevance of the concepts implemented. We have shown that the Virtual Desktop provides an immersive environment: it allows a natural way of working through flexible interactions to support design activity which is known as a high level activity. The concept of invisible computer supported by a mixed reality environment seems pertinent in the particular context of creative design tasks. This mixed reality paradigm is a strong way to support natural interaction, to insert the technology in current practices and environments in a smooth way and to support flexibility, multimodality and richness of interaction, invisible interface and augmented drawing, and a "soft" link between real world and virtual world.

Finally, the profession-centered approach and user-centered design offer a way to address the particularities of situations. They allow the design of adapted technologies, responding to real needs, useful in real situations. The simplicity and the specialization of the technology are the keys to the success of tomorrow's technologies, in opposition to the extreme complexity usually encountered in IT solutions [30]. Placing the users at the heart of the development is the required condition to design simple and specialised applications. 


\section{Bibliography}

1. Aliakseyeu, D. (2002) Direct manipulation interface for architectural design tools. Conference on Human Factors in Computing Systems, CHI '02 extended abstracts on Human factors in computing systems. Minneapolis, Minnesota, USA, pp. 536-537.

2. Aliakseyeu, D., Martens, J-B. (2001). Physical paper as the user interface for an architectural design tool. In: Proceedings of INTERACT 2001, Tokyo, Japan, July, pp. 680-681.

3. Bastien, C. \& Scapin, D. (1993). Ergonomic Criteria for the Evaluation of Human-Computer Interfaces. Rapport de l'INRIA : Institut National de Recherche en Informatique et en Automatique

4. Burkhardt, J-M., Détienne, F., Moutsingua-Mpaga, L., Perron, L. , Safin, S. Leclercq, P. (2008a) Multimodal collaborative activity among architectural designers using an augmented desktop at distance or in collocation. Proceedings of ECCE 2008 : European Conference on Cognitive Ergonomics. 16-19 September - Madeira, Portugal.

5. Burkhardt, J-M., Détienne, F., Moutsingua-Mpaga, L., Perron, L. , Safin, S. Leclercq, P. (2008b) Conception architecturale collaborative avec un «bureau augmenté » : une étude exploratoire de l'effet de la distance et de la co-localisation. Actes de SELF 2008 : 43ème congrès de la Société d'Ergonomie de Langue Française. 17-19 septembre 2008 - Ajaccio

6. Darses F., Mayeur A., Elsen C. et Leclercq P. (2008). Is there anything to expect from 3D views in sketching support tools? DCC'08, Proceedings of the 3rd International Conference on Design Computing and Cognition, Atlanta, USA.

7. Decortis, F., Safin, S. \& Leclercq (2005). A Role for External Representations in Architectural Design? The Influence of a Virtual Desk and an Early 3D View on the Design Activity. Proceedings of International Workshop on Understanding designers'05. Aix-en-Provence, France, October.

8. Dubois, E. (2001) Chirurgie Augmentée, un Cas de Réalité Augmentée; Conception et Réalisation Centrées sur l'Utilisateur. Thèse de doctorat, université Joseph Fourier, Grenoble I

9. Elsen C., Leclercq P. (2008). A sketching tool to support collaborative design. CDVE'08, 5th International Conference on Cooperative Design, Vizualisation and Engineering, Mallorca, Espagne.

10.Goel, V. (1995). Sketches of Thought. Bradford-MIT Press, Cambridge.

11.Goldschmidt, G. (1991). The Dialectics of sketching. Design Studies, Vol. 4, pp. 123-143.

12.Gross, M. (1996). The Electronic Coktail Napkin, Working with Diagrams. Design Studies, Vol. 17, pp.53-69

13.Gül, L. and Maher, M.L. (2006). The impact of Virtual Environments on Design Collaboration. In : 24th eCAADe Conference on Communicating Space(s), pp. 74-80.

14.Juchmes, R., Leclercq, P., Azar, S. (2004). A Multi-Agent System for Architectural Sketches Interpretation. Proceedings of Eurographics Workshop on Sketch-Based interfaces and Modeling, Grenoble, France, 53-62

15.Juchmes, R., Leclercq, P., Azar, S. (2005). A freehand sketch environment for architectural design supported by a multi-agent system. Special issue of Computers and Graphics on Calligraphic Interfaces, vol 29, num. 6, 905-915.

16.Kubicki S., Bignon J-C., Lotz J., Gilles Halin G., Elsen C. \& Leclercq P. (2008). Digital Cooperative Studio. ICE 2008 14th International Conference on Concurrent Enterprising, Special session ICT-supported Cooperative Design in Education, Lisboa, Espagne.

17.Kubicki S., Bignon J-C., Gilles Halin G., \& Leclercq P. (2008). Cooperative Digital Studio IT-supported cooperation for AEC students. CIB-W78 25th International Conference on Information Technology in Construction, Santiago de Chile, Chili.

18.Lebahar, J. (1983). Le dessin d'architecte. Simulation graphique et réduction d'incertitude. Paris : Editions Parenthèses.

19.Leclercq, P. (1999). Interpretative tool for architectural sketches. In Visual and Spatial Reasoning in Design, Gero, J., Tversky, B. (Eds.), Key Centre of Design Computing and Cognition, Sydney, Autralia, 69-80.

20.Leclercq, P. (2004). Invisible Sketch Interface in Architectural Engineering. Graphic recognition, recent avances and perspectives, Lecture Notes in Computer Science, LNCS 3088, Springer Verlag, Berlin, 353-363.

21.Leclercq, P. (2005) Le concept d'esquisse augmentée. Actes de SCAN'05 : Séminaire de Conception Architecturale Numérique. Paris, Ecole Nationale Supérieure d'Architecture de Paris-Val de Seine, France.

22.Leclercq, P. and Juchmes, R. (2002). The Absent Interface in Design Engineering. AIEDAM Artificial Intelligence in Engineering Design and manufacturing. Special issue: Human Computer Interaction in Engineering Contexts, Vol.16, n5, Cambridge university Press, November 2002.

23.Leclercq, P., Elsen, C. (2007). Le croquis synthé-numérique. Actes de SCAN 07 : Séminaire de conception architecturale numérique, Liège, Belgique. 
24.Leclercq P., Mayeur A., Darses F., (2007). Production d'esquisses créatives en conception digitale. Actes d'IHM'07, 17e conférence francophone sur l'Interaction Homme Machine, ICAM, Paris.

25.Mayeur A., Darses F., Leclercq P. (2008). Contributions of a 3D numerical environment for architectural sketches. Proceedings of the 8th International Symposium on Smart Graphics, Rennes, France. Lecture Notes in Computer Science, Springer.

26.Mayeur, A., Darses, F., Leclercq P., (2007a). Apports de la visualisation de maquettes virtuelles 3D en phase d'esquisse architecturale. Actes d'Epique'07, Congrès de la Société Française de Psychologie, UFR de Psychologie, Université de Nantes.

27. Mayeur A., Darses F. Leclercq P. (2007b). Evaluation ergonomique d'une tablette graphique d'aide à la conception architecturale. Actes de SELF'07, 42ème congrès de la Société d'Ergonomie de Langue Française, St-Malo.

28.McCall, R., E., Vlahos. \& Zabel, J. (2001). Conceptual Design as Hypersketching. In Proceedings of the 9th Int. Conference CAAD Futures, Kluwer Academic Publishers, Dordrecht, The Nederlands, pp. 285-298.

29.Norman, D. A. (1991). Cognitive artifacts. In Designing interaction: Psychology of the human-computer interface. J. M. Carroll (Ed.). Cambridge University Press, New York.

30.Norman, D.A. (1998). The invisible computer, MIT Press, Cambridge University Press, MA.

31.Norman, D.A. \& Draper, S.W.(1986). User Centered System Design. Hillsdale (NJ): Lawrence Erlbaum.

32.Pruitt, J. \& Grudin, J. (2003) Personas: practice and theory. Proceedings of the 2003 conference on Designing for user experiences. Pp 1 - 15. San Francisco, California.

33.Rabardel, P. (1995). Les Hommes et les Technologies. Approche Cognitive des Instruments Contemporains. Armand Colin, Paris.

34.Russell, D.M. Streitz, N. A., Winograd, T. (2005). Building diseappearing computers. A special issue of Communications of the ACM on The disappearing computer, a vision of computing that is truly unremarkable. vol. 48, num 3, 42-49.

35.Safin, S., Boulanger, C. \& Leclercq, P (2005) Premières évaluation d’un Bureau Virtuel pour un processus de conception augmenté. Proceedings of IHM 2005. Toulouse, France : ACM Press. pp. 107-11

36.Safin, S., Boulanger, C. \& Leclercq, P (2005). A Virtual Desktop's First evaluation for an Augmented Design Process. Proceedings of Virtual Concept 2005. Biarritz, France, November

37.Safin, S., Juchmes, R. \& Leclercq, P. (2008) Du crayon au stylo numérique : influences des interprétations numériques sur l'activité graphique en tâches de conception. Proceedings of IHM08 : Conférence francophone sur l'interaction homme-machine. Metz, septembre 2008.

38.Safin, S., Leclercq, P. \& Decortis, F. (2006) Understanding and supporting collaborative design using an horizontal tabletop display. Proceedings of IEA2006. $16^{\text {th }}$ World Congress on Ergonomics. Maastricht, The Netherlands, July.

39.Safin, S., Leclercq, P. \& Decortis, F. (2007) Impact d'un environnement d'esquisses virtuelles et d'un modèle 3D précoce sur l'activité de conception architecturale. Revue d’Interaction Homme-Machine. Vol 8, N², pp. 65-98.

40.Schuler, D. \& Namioka, A. (Eds) (1993). Participatory Design. Principles and Practices. Hillsdale (NJ): Lawrence Erlbaum.

41.Suwa, M., Gero, J.C. \& Purcell, T. (2000). Unexpected discoveries and S-invention of design requirements: important vehicles for a design process. Design Studies, Vol. 21, pp. 539-567

42.Suwa, M. \& Tverski, B. (1996). What Architects see in their Design Sketches : implications for design tools. In Proceedings of ACM Conf. On Human Factors in Computing Systems CHI'96, ACM Press, New York, pp. 191-192.

43.Vandamme, J.-F., Safin, S. \& Leclercq, P. (2007). Modalités d’interaction sur interface gestuelle : première évaluation des usages spontanés. Workshop sur les tables collaboratives, conférence IHM07 : Conférence francophone sur l'interaction homme-machine. Paris, novembre 2007

44.Verstijnen, I.M., van Leeuwen, C., Goldschmidt, G., Hamel, R., Hennessey, J.M. (1998). Sketching and creative discovery. Design Studies, vol. 19, num. 4, 519-546. 
